\title{
Research on attitude control strategy of single engine failure in the first-stage flight phase of new generation launch vehicle
}

\author{
Guorui Liao ${ }^{1, *}$, Jinjie $\mathrm{Liu}^{1}$, and Tianheng Dou ${ }^{1}$ \\ ${ }^{1}$ Xichang Satellite Launch Center, China
}

\begin{abstract}
The successful launching of the new generation of launch vehicle is of great practical significance to the development of China Space, and the cryogenic liquid engine commonly used in the launch vehicle has a certain probability of failures, which may affect the success or failure of rocket launching. In this paper, a Bolza problem optimization algorithm based on Radau pseudo-spectral method is proposed to solve the problem of single engine failure in the first-stage flight phase of the new generation launch vehicle by simulating fault injection and taking the attitude angle as optimal control quantity. The simulation results show that this method can effectively eliminate the influence of single engine failure on orbit injection accuracy, achieve the fault absorption of new generation launch vehicle engine, improve the fault tolerance of attitude control system, and further guarantee the system reliability.
\end{abstract}

\section{Introduction}

The new generation launch vehicle is an important means and strong guarantee for China to explore space resources, develop space technology and implement space strategy. It is also a milestone for China Space to become increasingly powerful. Every successful launching is of great practical significance to the development of China Space at present stage. The launching of the new generation launch vehicle is highly expected and attracts worldwide attention, which puts forward higher requirements for its launching success rate. Cryogenic liquid engine is commonly used in the power system design of the new generation launch vehicle, which has complicated structure and bad working environment, and has certain probability of flight failures, which may affect the success or failure of rocket launching. It is important to study the simulation modeling method, optimize the control strategy and reduce the impact of single engine failure on the flight of launch vehicle, which is of great significance to improve the system reliability of the new generation of launch vehicle.

At present, deep research has been carried out on attitude control technology of launch vehicle under engine failure in foreign countries. In 2012, both the Falcon-9 and the Delta-4 launch vehicles launched by the United States appeared single engine failure in the booster flight phase. Through attitude reconstruction and thrust compensation technology, the

*Corresponding author: 1ij91183269@163.com 
residual fuel was used to ensure the completion of the launch mission [1]. Domestic research on fault tolerance and reconfiguration control of launch vehicle research is relatively less. Meng Zhou [2] respectively based on the joint of rocket and booster to conduct control distribution and carried out relevant research on fault tolerance control method of stuck servo mechanism failures. Wang Zhixiang took the engine thrust drop failure as an example to study the on-line trajectory generation and attitude control system reconstruction technology of launch vehicle. Feng Hao, Li Xinming et al [3] carried out research on fault tolerance control of stuck servo mechanism failures, based on the idea of failure compensation. Huang Panxing [4] based on pseudo-spectral method and fixed point method to carry out fault tolerance reconfiguration design of launch vehicle under different failure modes of servo mechanism. The above examples are all aimed at the engine or servo mechanism failure in the booster flight phase. It is worth noting that there are multiple engines of rocketI and boosters working at the same time in the booster flight phase. If there is a single engine failure, we can achieve fault tolerance control through the method of control force compensation to redistribute the servo instruction. And for the new generation of launch vehicle, once appearing single engine failure in the first-stage flight phase, it means that only one engine could provide power. However, the quantity, position and swing direction of engine are very different from those in the booster flight phase, so we can't copy the fault tolerance reconfiguration design in previous research. At the same time, the rocket has got rid of the influence of dense atmosphere and arrived at a certain velocity and height in the first-stage flight phase. So if we can change the attitude control strategy in the case of the second stage working normally, it is possible for the rockets to enter orbit. Therefore, it is a realistic problem worthy of study to optimize the attitude control strategy in the case of single engine failure to guarantee the launching success rate of launch mission effectively.

\section{Dynamic modeling and optimization}

\subsection{Dynamics model of rocket in the first-stage flight phase}

At the beginning of the first-stage flight phase, the rocket has entered the vacuum flight through the dense atmosphere without aerodynamic force. It is mainly affected by the gravity of the earth and the thrust of engine. The deviation of engine parameters mainly includes the specific impulse, the deviation of propellant consumption per second, the lateral shift and deflection of the thrust action, etc. Ignoring the long-period of centroid motion, the liquid propellant swaying and elastic vibration of the rocket are not taken into account. The instantaneous equilibrium assumption is made during the flight process, and the selection of guidance parameters only includes two variables: pitch angle and yaw angle. Therefore, the differential equation of rocket dynamics can be written as [5]:

$$
\left\{\begin{array}{c}
\dot{x}=V_{x}, \dot{y}=V_{y}, \dot{z}=V_{z} \\
\dot{V}_{x}=-\frac{\mu x}{r^{3}}+\frac{F_{x}(t)}{m_{0}-\dot{m} t} \cos \varphi \cos \psi \\
\dot{V}_{y}=-\frac{\mu y}{r^{3}}+\frac{F_{y}(t)}{m_{0}-\dot{m} t} \sin \varphi \cos \psi \\
\dot{V}_{z}=-\frac{\mu z}{r^{3}}-\frac{F_{z}(t)}{m_{0}-\dot{m} t} \sin \psi
\end{array}\right.
$$


In the formula, the state variables $x, y, z, V_{x}, V_{y}, V_{z}, m$ respectively represent the position, velocity and mass of the rocket, and $\dot{m}$ is the propellant consumption per second of engine, which is usually a constant. The control variables $\varphi, \psi$ respectively represent the pitch program angle and yaw program angle of the rocket. $F(t)$ is the thrust of rocket engine.

\subsection{Optimization problem in the case of single engine failure}

If a single engine fails in the first-stage flight phase, the trajectory optimization problem can be regarded as the optimal control problem of Bolza form [6]. Specifically, the state variables (including the geocentric distance, longitude, latitude, velocity, orbit inclination and heading angle) are set to $\mathbf{x}(t) \in \mathbb{R}^{n}$. The control variables(attack angle and roll angle) are set to $\mathbf{u}(t) \in \mathbb{R}^{m}$. The time is $t$ and the initial time is $t_{0}$ when the terminal time is $t_{f}$. Then $u(t)$ is looked for to minimize the general Bolza type of objective function in the case of a series of constraint equations, which is:

$$
J=\Phi\left(\mathbf{x}\left(t_{0}\right), t_{0}, \mathbf{x}\left(t_{f}\right), t_{f}\right)+\int_{t_{0}}^{t_{f}} g(\mathbf{x}(t), \mathbf{u}(t), t) d t
$$

The motion equation can be written as:

$$
\frac{d \mathbf{x}}{d t}=\mathbf{f}(\mathbf{x}(t), \mathbf{u}(t), t)
$$

The process constraint can be written as:

$$
\mathbf{C}(\mathbf{x}(t), \mathbf{u}(t), t) \leq 0
$$

The endpoint constraint can be written as:

$$
\phi\left(\mathbf{x}\left(t_{0}\right), t_{0}, \mathbf{x}\left(t_{f}\right), t_{f}\right)=0
$$

Due to the need of follow-up work, the time variable is transformed. The time interval $t \in\left[t_{0}, t_{f}\right]$ is divided into $K$ subintervals $\left[t_{k-1}, t_{k}\right],(k=1, \cdots, K)$, and $t_{0}<t_{1}<\cdots<t_{K}=t_{f}$. Then, $t \in\left[t_{k-1}, t_{k}\right]$ of each subinterval is converted to $\tau=[-1,+1]$ through the following equations.

$$
\begin{aligned}
\tau & =\frac{2 t-\left(t_{k}+t_{k-1}\right)}{t_{k}-t_{k-1}}\left(t_{k-1}<t_{k}\right) \\
\frac{d \tau}{d t} & =\frac{2}{t_{k}-t_{k-1}},(k=1, \cdots, K)
\end{aligned}
$$

The state variable and control variable of each subinterval are $\mathbf{x}^{k}(\tau)$ and $\mathbf{u}^{k}(\tau)$ respectively.

Equations (1) (5) can be converted into: 


$$
\begin{gathered}
J=\Phi\left(\mathbf{x}^{(1)}(-1), t_{0}, \mathbf{x}^{(K)}(+1), t_{f}\right)+\sum_{k=1}^{K} \frac{t_{k}-t_{k-1}}{2} \int_{-1}^{+1} g\left(\mathbf{x}^{k}(\tau), \mathbf{u}^{k}(\tau), \tau ; t_{k-1}, t_{k}\right) d \tau \\
\frac{d \mathbf{x}^{(k)}(\tau)}{d \tau}=\frac{t_{k}-t_{k-1}}{2} \mathrm{f}\left(\mathbf{x}^{k}(\tau), \mathbf{u}^{k}(\tau), \tau ; t_{k-1}, t_{k}\right) \\
C\left(\mathbf{x}^{k}(\tau), \mathbf{u}^{k}(\tau), \tau ; t_{k-1}, t_{k}\right) \leq 0 \\
\phi\left(\mathbf{x}^{(1)}(-1), t_{0}, \mathbf{x}^{(K)}(+1), t_{f}\right)=0
\end{gathered}
$$

Since the state variable is continuous, the boundary of each interval should be connected, which is $\mathbf{x}\left(t_{k}^{-}\right)=\mathbf{x}\left(t_{k}^{+}\right)$, where $k=1, \cdots, K-1$.

\section{Solving nonlinear problems by Radau pseudo-spectral method}

As shown above, the continuous time optimal problem needs to be parameterized in the time domain and transformed into the nonlinear programming NLP problem, and then the numerical optimal solution of the NLP problem is obtained by numerical method.

Analyzing the three attitude angles of the rocket under normal flight, it is found that the main attitude control strategy is reflected in the pitch angle. After the initial roll (booster flight phase), the yaw angle and roll angle almost remain around 0 . In order to simplify the problem and reduce the coupling effect of attitude control channel, this paper only takes the pitch angle as the attitude control variable.

When a single engine fails, the existing attitude control strategy will constantly adjust the attitude of the rocket, and a large amount of thrust is spent on frequent attitude adjustment, which makes the height and velocity of the rocket not up to the requirements. The load cannot be put into the designated orbit even under the normal operation of the second-stage flight phase. Therefore, this paper proposes an attitude control strategy. After detecting a significant step of acceleration, the rocket immediately flies at a fixed pitch angle to study the possibility of entering orbit. Fig. 1 shows the height of rocket after the end of the first-stage flight phase when different pitch angles are set at the preset failure point. It can be seen that when the fixed pitch angle is greater than $20^{\circ}$, the rocket height will not drop, or the normal orbit can be achieved after the second-stage flight phase. Therefore, the control variable can be further simplified to a fixed pitch angle. Since the fixed pitch angles of the first and second stage may be different, the control variable can be set as a piecewise constant function.

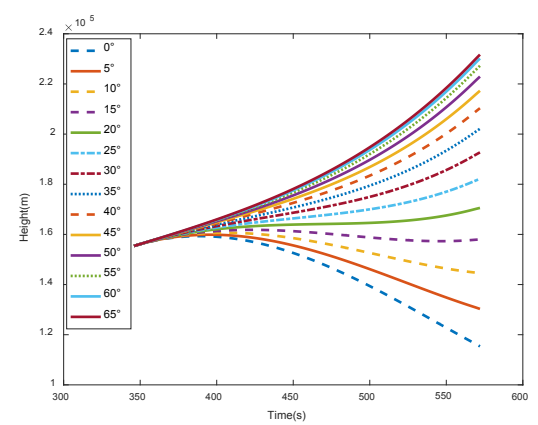

Fig. 1. Height in the first-stage flight phase with different pitch angles. 
At this case, the state variables only need to be discretized. By discretizing the time on the finite nodes and discretizing the state variables on the time nodes, the interpolation function is used for the interpolation approximation of state variables between the nodes to transform differential state equation constraints into algebraic equation constraints, and the attitude control optimization problem can finally be transformed into a general nonlinear programming problem. As one of the collocation methods, Radau pseudo-spectral method [7] has attracted much attention in recent years. It has been applied in theoretical fields such as solving the optimal control problem quickly and optimizing aircraft trajectory on-line. As described above, the whole time interval has been divided into many subintervals, and the state variables of each subinterval can be approximately expressed by polynomial of $N_{k}$ degree:

$$
\mathbf{x}^{(k)}(\tau) \approx \mathbf{X}^{(k)}(\tau)=\sum_{j=1}^{N_{k}+1} \mathbf{X}_{j}^{(k)} L_{j}^{(k)}(\tau) \quad L_{j}^{(k)}(\tau)=\prod_{\substack{l=1 \\ i \neq j}}^{N_{k}+1} \frac{\tau-\tau_{l}^{(k)}}{\tau_{j}-\tau_{l}}
$$

where $\tau=[-1,+1], \quad L_{j}^{(k)}(\tau),\left(j=1, \cdots, N_{k}+1\right)$ is polynomial function of Lagrange interpolation, $\left(\tau_{1}^{(k)}, \cdots, \tau_{N_{k}}^{(k)}\right)$ is the LGR (Legendre-Gaus-Radau) discrete points in each subinterval, $\tau_{N_{k}+1}^{(k)}=+1$ does not belong to LGR discrete points. By differentiating $\mathbf{X}^{(k)}(\tau)$, we get the following results:

$$
\frac{d \mathbf{X}^{(k)}(\tau)}{d \tau} \equiv \dot{\mathbf{X}}^{(k)}(\tau)=\sum_{j=1}^{N_{k}+1} \mathbf{X}_{j}^{(k)} \dot{L}_{j}^{(k)}(\tau)
$$

Using LGR integral, the objective function can be approximated as:

$$
J \approx \Phi\left(\mathbf{X}_{1}^{(1)}, t_{0}, \mathbf{X}_{N_{k}+1}^{(K)}, t_{K}\right)+\sum_{k=1}^{K} \sum_{j=1}^{N_{k}} \frac{t_{k}-t_{k-1}}{2} \omega_{j}^{(k)} g\left(\mathbf{X}_{j}^{(k)}, \mathbf{U}_{j}^{(k)}, \tau_{j}^{(k)} ; t_{k-1}, t_{k}\right)
$$

where $w_{j}^{(k)}\left(j=1, \cdots, N_{k}\right)$ is the LGR weighted term, $\mathbf{U}_{j}^{(k)}$ is the approximation of the control variable at the LGR point in the subinterval $k, \mathbf{X}_{1}^{(1)}$ is the approximation of $\mathbf{x}\left(t_{0}\right)$ and $\mathbf{X}_{N_{k}+1}^{(K)}$ is the approximation of $\mathbf{x}\left(t_{f}\right)$.

The discrete form of motion equation (3) at the LGR point is:

$$
\sum_{j=1}^{N_{k}+1} \mathbf{X}_{j}^{(k)} D_{i j}^{(k)}-\frac{t_{k}-t_{k-1}}{2} \mathbf{f}\left(\mathbf{X}_{i}^{(k)}, \mathbf{U}_{i}^{(k)}, \tau_{i}^{(k)} ; t_{k-1}, t_{k}\right)=0,\left(i=1, \cdots, N_{k}\right)
$$

where

$$
D_{i j}^{(k)}=\dot{L}_{j}^{(k)}\left(\tau_{i}\right),\left\{\begin{array}{l}
\left(i=1, \cdots, N_{k}\right) \\
\left(j=1, \cdots, N_{k}+1\right) \\
(k=1, \cdots, K)
\end{array}\right.
$$

The discrete form of process constraint (4) at the LGR point is: 


$$
\mathbf{C}^{(k)}\left(\mathbf{X}_{i}^{(k)}, \mathbf{U}_{i}^{(k)}, \tau_{i} ; t_{k-1}, t_{k}\right) \leq \mathbf{0},\left(i=1, \cdots \cdots, N_{k}\right)
$$

The discrete form of endpoint constraint (5) at LGR point is:

$$
\boldsymbol{\phi}\left(\mathbf{X}_{1}^{(1)}, t_{0}, \mathbf{X}_{N_{K}+1}^{(K)}, t_{K}\right)=\mathbf{0}
$$

Through a series of numerical approximation methods mentioned above, the continuous Bolza problem is finally transformed into a nonlinear programming problem, that is, the state variables and control variables at each LGR point are obtained, and the performance objective function (14) is minimized under the condition that the motion equation (3), process constraint (4) and endpoint constraint (5) are satisfied. In this paper, sequential quadratic programming (SQP) algorithm is used as a solver to solve the above nonlinear programming problems.

In each subinterval $k \in[1, \ldots, K], L$ sampling points $\left(\bar{\tau}_{1}^{(k)}, \ldots, \bar{\tau}_{L}^{(k)}\right) \in[-1,1]$ are set to calculate the accuracy of each subinterval. Then, the error calculated by the discrete motion equation and process constraint equation of each subinterval can be expressed as:

$$
\begin{gathered}
\left|\dot{\mathbf{X}}^{(k)}\left(\bar{\tau}_{l}^{(k)}\right)-\frac{t_{k}-t_{k-1}}{2} \mathbf{f}^{(k)}\left(\mathbf{X}_{l}^{(k)}, \mathbf{U}, \bar{\tau}_{l}^{(k)} ; t_{k-1}, t_{k}\right)\right| \\
\mathbf{C}_{l}^{(k)}\left(\mathbf{X}_{l}^{(k)}, \mathbf{U}, \bar{\tau}_{l}^{(k)} ; t_{k-1}, t_{k}\right)=\boldsymbol{b}_{l}^{(k)}
\end{gathered}
$$

If $\boldsymbol{e}_{l}^{(k)}$ and $\boldsymbol{b}_{l}^{(k)}$ are both less than $\varepsilon_{d}$, then the current state variable and control variable are the approximate solutions of the optimal control; if any value of $\boldsymbol{e}_{l}^{(k)}$ and $\boldsymbol{b}_{l}^{(k)}$ is greater than $\varepsilon_{d}$, it indicates that the current meshing does not meet the accuracy requirements, and it is necessary to increase the order of Lagrange polynomial or the number of time segments in each subinterval. In the absence of process constraints, $\boldsymbol{b}_{l}^{(k)}$ is negative, which is always less than $\varepsilon_{d}$; when process constraints exist, $\boldsymbol{b}_{l}^{(k)}$ may be positive. If the value exceeds $\varepsilon_{d}$, it indicates that the current calculation accuracy does not meet the requirements and the grid needs to be reconstructed.

1) Determination of the reconstruction method

Suppose the curvature of the state component $X_{m}^{(k)}(\tau)$ of $m$ in the subinterval of $k \in[1, \ldots, K]$ as:

$$
\kappa^{(k)}(\tau)=\frac{\left|\ddot{X}_{m}^{(k)}(\tau)\right|}{\left|\left[1+\dot{X}_{m}^{(k)}(\tau)^{2}\right]^{3 / 2}\right|}
$$

Supposing that the $\kappa_{\max }^{(k)}$ and $\tilde{\kappa}^{(k)}$ respectively are the maximum and average values of $\kappa^{(k)}(\tau)$ calculated by all the state variables in the subinterval of $k$, and a judgment $r_{k}=\kappa_{\max }^{(k)} / \tilde{\kappa}^{(k)}$ is introduced. If $r_{k}<r_{\max }$, then the order of polynomials in each subinterval needs to be increased; if $r_{k}>r_{\max }$, then more subintervals need to be divided. 
2) Determination of the Lagrange polynomial order in each subinterval

Supposing that the matching points in each subinterval before and after updating are $N_{k}^{-}$and $N_{k}^{+}$respectively, then the functional relationship between the error tolerance $\varepsilon_{d}$ and the maximum error $e_{\max }^{(k)}$ before and after updating can be expressed as:

$$
N_{k}^{+}=N_{k}^{-}+\operatorname{ceil}\left(\log _{10}\left(e_{\max }^{(k)}\right)-\log _{10}\left(\varepsilon_{d}\right)\right)+X
$$

where $X$ is an arbitrary integer, which is used to control the growth of matching points in each interval.

3) Determination of the number of the segments divided again in each subinterval

Suppose the number of the segments divided again in the subinterval of $k$ as $n_{k}$, then:

$$
n_{k}=Y \operatorname{ceil}\left(\log _{10}\left(e_{\max }^{(k)}\right)-\log _{10}\left(\varepsilon_{d}\right)\right)
$$

where $Y$ is an arbitrary integer, which is used to control the growth of the segments number.

Supposing that the minimum order of the Lagrange polynomial is $m$ in each subinterval(equal to the number of matching points in each subinterval), and then adaptive updating can be carried out according to the process shown in Fig. 2:

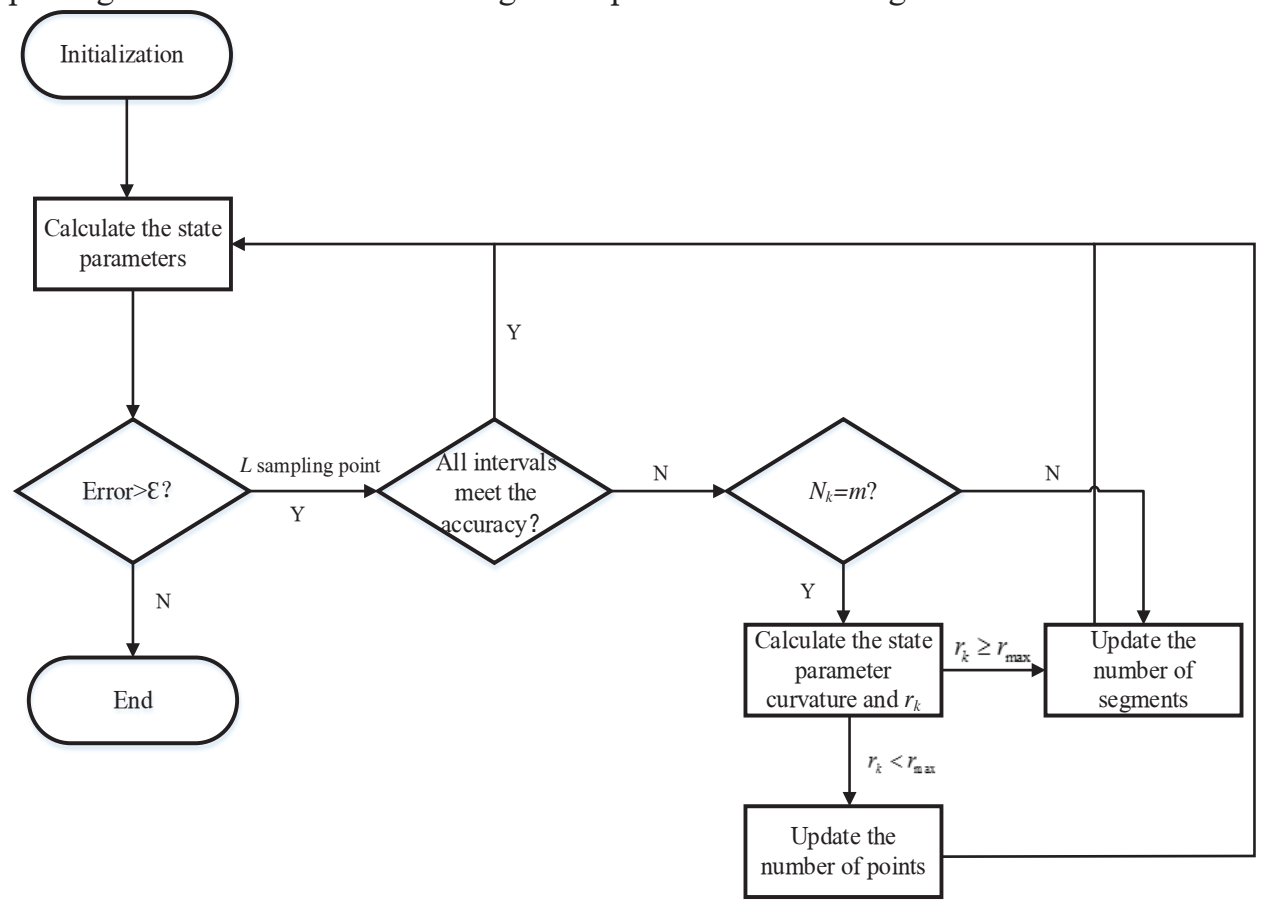

Fig. 2. HP adaptive updating process.

\section{Fault simulation and strategy optimization}

Matlab platform is used to simulate the flight process of the new generation launch vehicle. In the case of single engine failure, the thrust of rocket decreases and the attitude becomes 
unstable, which is mainly reflected in the abrupt drop of total velocity and the divergence of attitude angle in the data. Fig. 3 shows the normal data and fault data obtained from the simulation based on the existing attitude control strategy. It can be seen that when a single engine fails in the first-stage flight phase (the red frame area), the total acceleration data occurs an instantaneous step immediately, and the attitude angle data oscillates at the same time. The amplitude of the oscillation changes from small to large, and finally diverges. Considering the timeliness and fault tolerance of the attitude control strategy, this paper selects the instantaneous large step of the total acceleration as the trigger condition of the optimal attitude control strategy.
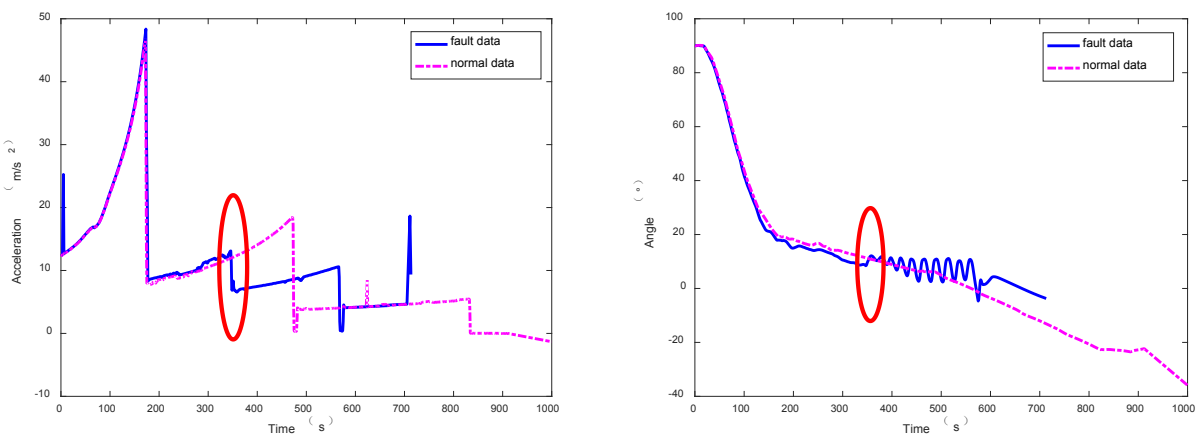

Fig. 3. Simulation of fault data.

Fig. 1 shows the flight with fixed pitch angle in the first-stage flight phase, and Fig. 4 shows the orbit height after completing the second-stage flight phase with fixed pitch angle. It can be seen that, as mentioned above, when the fixed pitch angle is greater than $20^{\circ}$, there will be no height drop in the first-stage flight phase. However, the larger the fixed pitch angle is, the lower the orbit height will be after the second-stage flight phase. Therefore, it is necessary to optimize the calculation of the pitch angle.

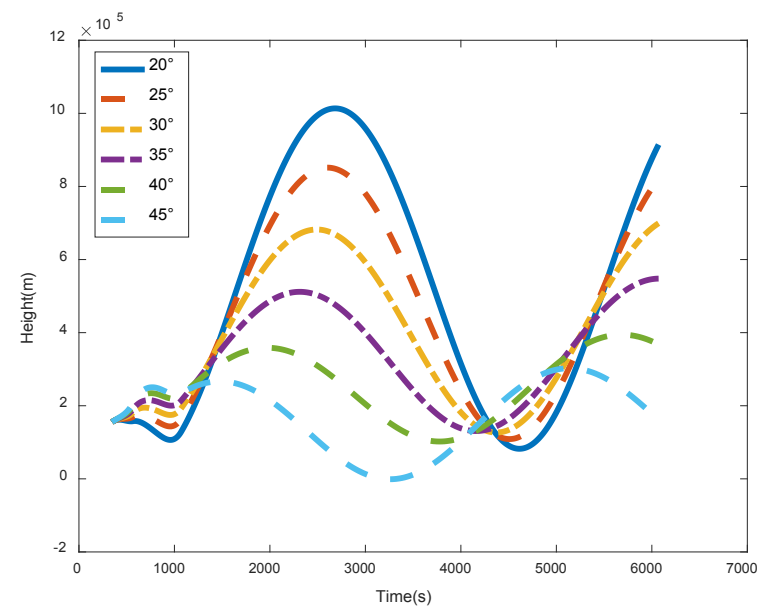

Fig. 4. Height of the second-stage flight phase with different pitch angles.

Taking the orbit accuracy (height and velocity selected in this paper) as the final optimization objective, the position and velocity as the initial conditions when the total acceleration occurs an instantaneous large step, the fixed pitch angle as control variable, and ignoring the adjusting time of the servo mechanism in response to the attitude control 
instruction and the rocket rolling caused by single engine failure, the Bolza optimal problem is solved by pseudo-spectral method.

The optimization results show that if a single engine fails at the time point shown in Fig. 3 , the load can be sent into the designated orbit if the first stage maintains a fixed pitch angle of $23.47^{\circ}$ and the second stage maintains a fixed pitch angle of $-29.58^{\circ}$. According to the target results shown in Fig. 1 and Fig. 4, it is known that if the load could be sent into the designated orbit, the pitch angle of the first stage should be greater than $20^{\circ}$ and less than $45^{\circ}$. So the calculated results of pitch angle are consistent with the target results. The trajectories obtained according to the existing strategy and the optimized attitude control strategy are shown in Fig. 5(a), and Fig. 5(b) shows the complete orbit height. Obviously, the problem of rocket launching failure caused by single engine failure can be solved through strategy optimization, and the system fault tolerance of rocket can be improved.

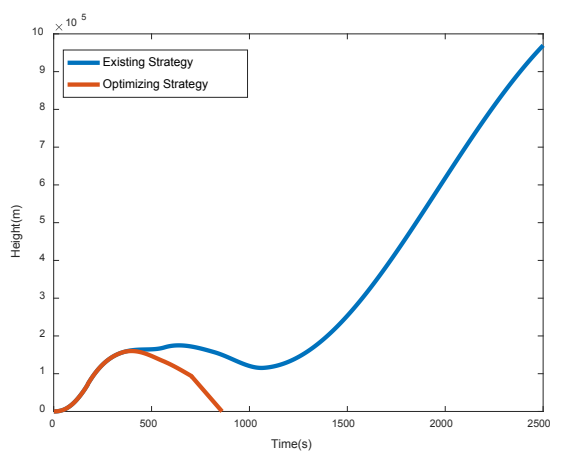

(a)

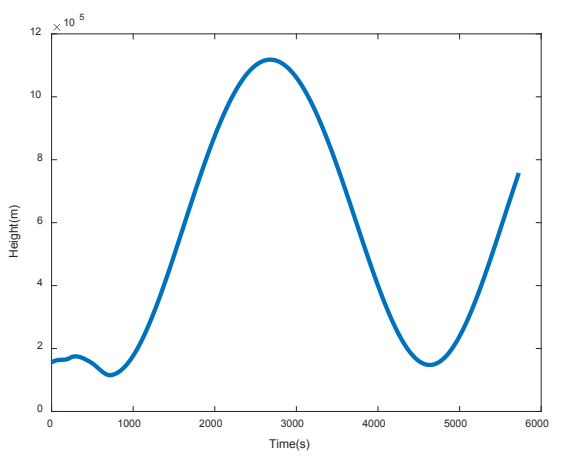

(b)

Fig. 5. Orbit height after optimizing attitude control strategy.

Another significance of studying the fault tolerance strategy of single engine failure in the first-stage flight phase is to decide whether to start safety self-destruct. Fig. 6 shows the final orbit height reached with optimal pitch angle when the single engine failure occurs at different time. It can be seen that from 300 s to the end of the first-stage flight phase, the load can be sent into the circle orbit above $100 \mathrm{~km}$ through the optimization of attitude control strategy.

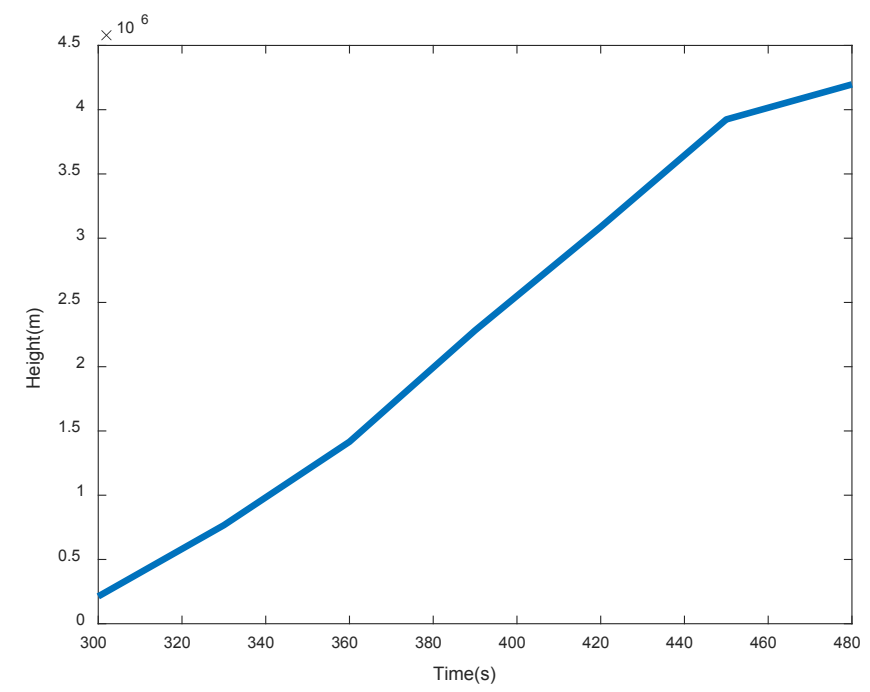

Fig. 6. Optimal orbit height reached when faults occur at different time. 


\section{Conclusion}

Aiming at the problem of single engine failure in the first-stage flight phase of the new generation launch vehicle, a Bolza problem optimization algorithm based on Radau pseudospectral method is proposed in this paper. By discretizing the time on the finite nodes and discretizing the state variables on the time nodes, the interpolation function is used for the interpolation approximation of state variables between the nodes to transform differential state equation constraints into algebraic equation constraints, and the attitude control optimization problem can finally be transformed into a general nonlinear programming problem. The simulation results show that this method can effectively eliminate the influence of single engine failure on orbit injection accuracy, achieve the fault absorption of new generation launch vehicle engine, improve the fault tolerance of attitude control system, and further guarantee the system reliability. It provides a feasible idea and beneficial reference for the attitude control strategy optimization and security control scheme in actual rocket test and launch mission.

\section{References}

1. Qin Xudong, Long Lehao, Rong Yi. The Achievement and Future of China Space Transportation System [J] Journal of Deep Space Exploration, 3 (4) : 315(2016).

2. Meng Zhou. Fault Tolerant Control for Large Strapon Launch Vehicle's Servomechanism[D]. Changsha: Natioanal University of Defense Technology(2013).

3. Feng Hao, Li Xinming, Pan Hao. On Reconstruction Technologies of Thrusting Force For Heavy Thrust Launch Vehicles[J]. Manned Spaceflight, 018(006):75-79(2012).

4. Huang Panxing.Research on Reconfigurable Control Systems Design of Heavy Launch Vehicle[D].Harbin: Harbin Institute of Technology(2012).

5. Zhu Haiyang, Wu Yansheng, Chen Yu, Yang Yunfei, Xu Lijie. A Neural Network Fault-Tolerant Control Method for Launch Vehicles with Thrust Decline[J]. Aerospace Control, 2019,37(4):3-9

6. A, A. J. M. Ferreira, and G. E. F. B . Computation of natural frequencies of shear deformable beams and plates by an RBF-pseudospectral method. Computer Methods in Applied Mechanics and Engineering, 196. 1-3(2006):134-146..

7. Tian, Bailing, and Q. Zong . Optimal guidance for reentry vehicles based on indirect Legendre pseudospectral method. Acta Astronautica 68.7-8(2011):1176-1184. 